\title{
Image analysis algorithm for detection and measurement of Martian sand grains
}

\author{
Joanna Kozakiewicz ${ }^{1,2}$ (D) \\ Received: 1 July 2017 / Accepted: 17 January 2018 / Published online: 23 January 2018 \\ (C) The Author(s) 2018. This article is an open access publication
}

\begin{abstract}
Image analysis methods are commonly employed to determine the size and shape of particles. Although commercial and noncommercial tools enable detection and measurement of grains from images, they do not provide good results in the case of images acquired during extensive in situ Martian investigations. Within the confines of the Mars Exploration Rover (MER) mission and the Mars Science Laboratory (MSL) mission thousands of images of sand grains were captured, and hitherto, they are the only source of ground-truth data on Martian sand particles. Therefore, a new approach is proposed to analyze such images. The semiautomatic algorithm allows fast detection and measurement of the size and shape of Martian grains from images obtained by the Microscopic Imager (MI) and the Mars Hand Lens Imager (MAHLI). The method was evaluated on 76 images of terrestrial and Martian deposits. The results for the terrestrial samples were compared to those from sieve analysis, as well as with ImageJ and Malvern Morphologi G3 systems. The method provides similar results to those from the other methods. It does not have any limitation on the size of grains, and permits separation of touching particles.
\end{abstract}

Keywords Image analysis $\cdot$ Algorithm $\cdot$ Sand grains $\cdot$ Mars

\section{Introduction}

Aeolian processes play a significant role in shaping the present surface of Mars. To obtain information on aeolian transport we need to bring together data about aeolian bedforms and the materials that they are composed of. Therefore, we need to measure chemical, mineralogical and physical properties of Martian deposits. Among physical properties important for aeolian transport are the size and the shape of transported grains. To study mechanisms of aeolian transport on Mars, it is then very crucial to determine statistical characteristics of deposits, and to investigate correlations between their various parameters, such as the mean diameter,

Communicated by: H. A. Babaie

Joanna Kozakiewicz

j.kozakiewicz@uj.edu.pl

1 Institute of Geography and Spatial Management, Jagiellonian University, Krakow, Poland

2 Astronomical Observatory, Jagiellonian University, 171 Orla St., 30-244 Krakow, Poland elongation or sorting of grains (Friedman 1967; Moiola and Weiser 1968; Visher 1969; Borowka 1980).

Terrestrial aeolian deposits are mainly studied using sieve and laser diffraction analysis, but on Mars, such methods cannot be applied yet. There is, however, another option as various Martian sediments are clearly visible in images acquired during the rovers' missions. From such images, morphological parameters of deposits can be estimated.

The rovers of the MER (Mars Exploration Rover) mission: Opportunity and Spirit, located in Gusev Crater and Meridiani Planum, respectively, were equipped with the MI (Microscopic Imager), a high-resolution camera. A camera of still higher resolution, the MAHLI (Mars Hand Lens Imager), was mounted on the MSL (Mars Science Laboratory) rover, Curiosity, investigating Gale Crater. These rovers acquired thousands of images in which individual sand grains can be resolved.

The MI and MAHLI images were taken under very different settings from laboratory conditions. An example of an image obtained in laboratory conditions can be seen in Fig. $1 \mathrm{c}, \mathrm{d}$, whereas a detailed image of the Martian surface is shown in Fig. 1a,b. For both Martian and terrestrial images, we would like to have a simple tool that enables the study of sand-sized material. Therefore, this work presents a semiautomatic method, which allows studying the shape and size 

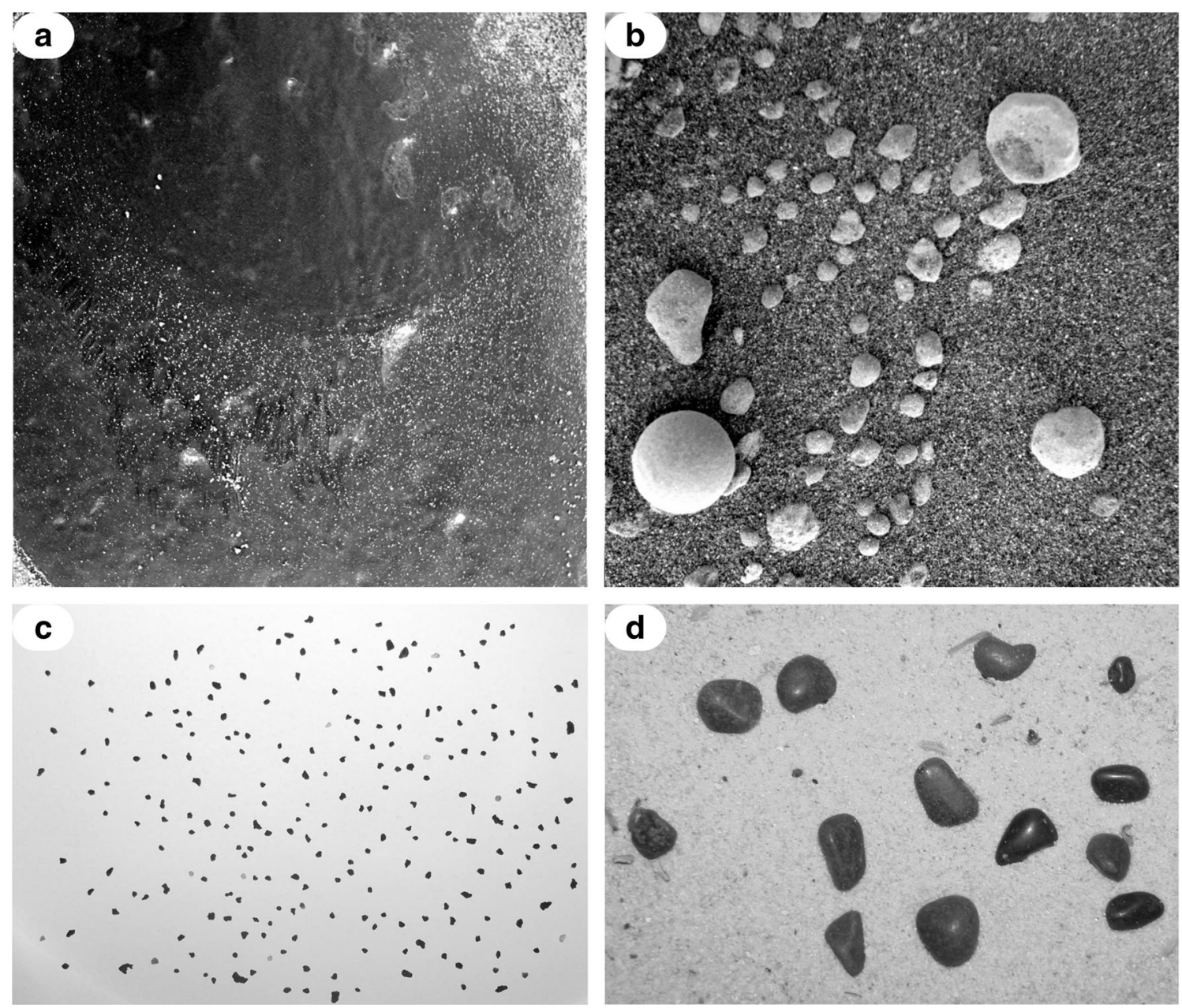

Fig. 1 Images of deposits. a Dust aggregates from Gusev Crater (sol 79, image ID: 2M133382890EFF2232P2977M2M1). b Spherules from Meridiani Planum (sol 19, image ID: 1M129869918EFF0338

of grains from images. It is based on digital image processing and analysis techniques.

\section{MI and MAHLI images}

The MI on both MER rovers operated identically, so that data processing in these two cases was done in the same way. The MI images are $1024 \times 1024$ pixels in size with $31 \mu \mathrm{m}$ per pixel at best focus, resulting in around $31 \mathrm{~mm}$ frame width (Herkenhoff et al. 2003). Pixel size of the MAHLI images is not constant as the camera can focus from $2.1 \mathrm{~cm}$ to infinity. Knowing the distance between the front lens element and the image target, the physical size of the image can be calculated. The relationship between pixel size, $p$ (in $\mu \mathrm{m} / \mathrm{pixel}$ ), and working distance, $w$ (in $\mathrm{cm}$ ), is given by the equation (Minitti et al. 2013):

$p=6.9001+(3.5201 \times w)$.
P2953M2M1). c Dark sand grains from Grand Falls dune field, Arizona, US. d Small pebbles from Iceland on fine desert sand

Martian images are corrected (Herkenhoff et al. 2006; Edgett et al. 2012) using additional calibrating images as flat field, dark and bias frames. Such processing improves image quality, however, it does not remove uneven illumination or background noise. Illumination in a few Martian images varied beyond any satisfactory correction, and it became necessary to crop the images manually. In these cases, the further processing was done solely to the fragment of the image that was the most evenly illuminated and represented the greatest number of grains.

The resolution of the MI images does generally not allow distinguishing grains smaller than $0.15 \mathrm{~mm}$. Studying smaller particles was possible only in some cases. Although the main aim of this work is to study sand grains, grains larger than $2.0 \mathrm{~mm}$ were analyzed also.

Different types of grains can be differentiated in the Martian images (Geissler et al. 2008). Fine-grained material composed of dust aggregates of diameters $<0.15 \mathrm{~mm}$ is present in almost all images (Fig. 1a,b). Spherules or their 
fragments are visible in many images from the Opportunity rover (Fig. 1b) (Squyres et al. 2004). These grains are generally a few millimeters in diameter, and they are usually not touching one another when imaged on the surface. Grains found in ripple crests and dune fields are smaller than spherules. They are well sorted and densely packed. Individual grains smaller than $1 \mathrm{~mm}$, but larger than $0.15 \mathrm{~mm}$, are clearly visible only in a few images.

\section{Methodology}

\section{Background}

Several different methods in image analysis were proposed to acquire information on particles from images. An approach in principle based on sieve analysis is the granulometry method (Matheron 1967). The method uses a series of morphological opening or closing operations, and it provides only some information on the size of grains passing through the given structuring element. This technique does not allow studying the shape of grains. To obtain information on morphological parameters of individual particles, an image segmentation technique, enabling the detection of grain boundaries, must be applied (Fu and Mui 1981). This approach often allows the separation of overlapping or touching grains.

A granulometry technique with opening operations was employed to the MI images (Lira et al. 2010), yet it resulted solely in a rough estimation of granulometric curves.

The estimation of shape and size of Martian grains has been done manually in a number of publications (Weitz et al. 2006; Jerolmack et al. 2006; Yingst et al. 2008; Sullivan et al. 2008; Minitti et al. 2013; Yingst et al. 2013). Manual methods, however, are very time-consuming, tedious, and are not practical as the number of images steadily increases.

The work of Karunatillake et al. (2014) presents a semiautomatic method for segmenting the Martian images, especially those obtained by the MI instrument, using image processing techniques. This method is based on two procedures: binarization and watershed segmentation. The authors study the foreground (defined in their work as grains position closer to the imager) and background of an image separately. They use binarization technique to establish particles in the foreground, yet this technique does not allow separation of touching grains. If such particles are present in the foreground, binarization will not give a precise result. Due to the large number of operations, and the number of necessary parameters that must be established during the segmentation process the method is also quite complicated.

Therefore, a simple tool dedicated to analyzing Martian images is presented in this work. It is based on the PADM (Particle Detection and Measurement) algorithm, which enables fast detection and measurement of individual grains, and also provides statistical results. The PADM algorithm uses a set of image processing operations associated with filtering, segmentation and extraction. The algorithm was originally implemented into Wolfram Mathematica, but it can be implemented into any other environment, such as IDL or Matlab.

Although it is possible to establish automatic parameters for each group of Martian images, the accuracy of the results can be improved significantly with some human assessment. Therefore, the approach presented here is semi-automatic.

\section{PADM algorithm}

The algorithm employs segmentation techniques for object detection. Hence, in the initial step, it is necessary to determine the foreground and the background of studied images.

Images of deposits can be divided into four main groups according to the foreground-background relationship: a) grains can be brighter than their surroundings and smaller than the background irregularities (Fig. 1a), b) grains can be brighter than their surroundings and larger than the background irregularities (Fig. 1b), c) grains can be darker than their surroundings and smaller than the background irregularities (Fig. 1c), d) grains can be darker than their surroundings and larger than the background irregularities (Fig. 1d).

Almost all particles observed in the Martian images are bright. However, before using the algorithm, we have to decide whether objects we would like to detect are characterized by higher (Fig. 1a,b) or lower (Fig. 1c,d) values relative to their surroundings.

If the grains are smaller than the background irregularities (Fig. 1a,c), the background can be easily removed, but if grains are larger or similar to surface irregularities (Fig. 1b,d), then the background often cannot be subtracted without some reduction of the foreground. On Earth, even during a field experiment, we can easily take images of material placed on some plain background (Fig. 1c), for example on a piece of paper. However, many Martian sand grains are located on a deep layer of fine material, which creates non-uniform, noisy background (Fig. 1b). Furthermore, some Martian images do not display a clearly visible background, but instead contain many touching particles (Fig. 2). Since the irregularities associated with such a "background" are similar to the size of particles, this kind of images should be processed like those with a noisy background.

To distinguish between a plain and a noisy background, an initial automatic procedure, called background procedure $(B G)$ is applied to the algorithm. It is based on calculating the volume of the studied image, $f$, after its filtering and binarization. The filtering is done by the top-hat transform $\operatorname{THT}_{R}(f)$ (for brighter grains) or the bottom-hat transform $B H T_{R}(f)$ (for darker grains) with a structuring element in a form of a disk of a range $R$. Then the output image, $f_{1}$, is binarized with the threshold operator $T_{[\alpha, \beta]}$ with the lower and upper thresholds: $\alpha$ and $\beta$. The threshold operator 


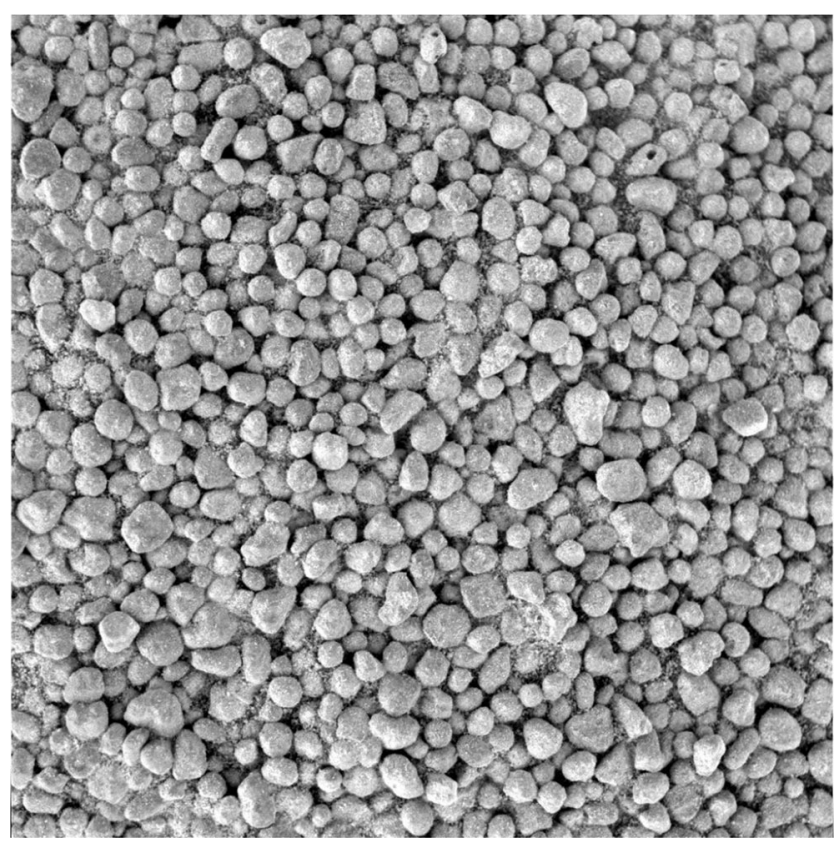

Fig. 2 Particles from Meridiani Planum (sol 368, image ID: 1M160851916EFF42D9P2997M2M1)

converts the input image $f_{1}$ into a binary image whose pixels values are either 0 or 1 . Next, the volume of the image $f_{2}$ is computed: $V_{B F(R)}=\sum_{p \in D} f_{2}(p)$, where $p$ - the image pixel, $D$
- the image domain. Finally, the calculated volume is normalized to the volume $V$ of the input image $f: V_{N(R)}=V_{B F(R)} / V$, where: $V=\sum_{p \in D} f(p), V_{N(R)}$ is the normalized volume. The procedure starts from $R=1$, and ends when it finds the first minimum of $V_{N(R)}$, i.e. when $R=M+1$, for which $V_{N(M)}<$ $V_{N(M+1)}$ (where: $M=1,2, \ldots$, is much smaller than the size of grains). The $B G$ procedure returns a logical value, which is true if the value of the first minimum of $V_{N(R)}$ is larger than an empirically determined critical value for a given set of images. For the Martian and terrestrial images studied in this work, the Otsu's threshold (Otsu 1979) was used, and the background was treated as noisy when the value of the first minimum of $V_{N(R)}$ was larger than 0.06 .

The PADM algorithm employs three different techniques of grain detection, as one of them may work better than another with a particular image. These three techniques are called after their main procedures: binarization, edge detection and watershed segmentation. For all images with removable background, all approaches can be used. If the reduction of the background is not possible but the grains are separated, often the most efficient technique is the edge detection. In cases with many touching particles, the marker-controlled segmentation processing sequence (watershed) can be applied.

All these techniques consist of four steps. Each step is described below and the scheme of the whole algorithm is presented (Fig. 3).
Fig. 3 The scheme of the PADM algorithm

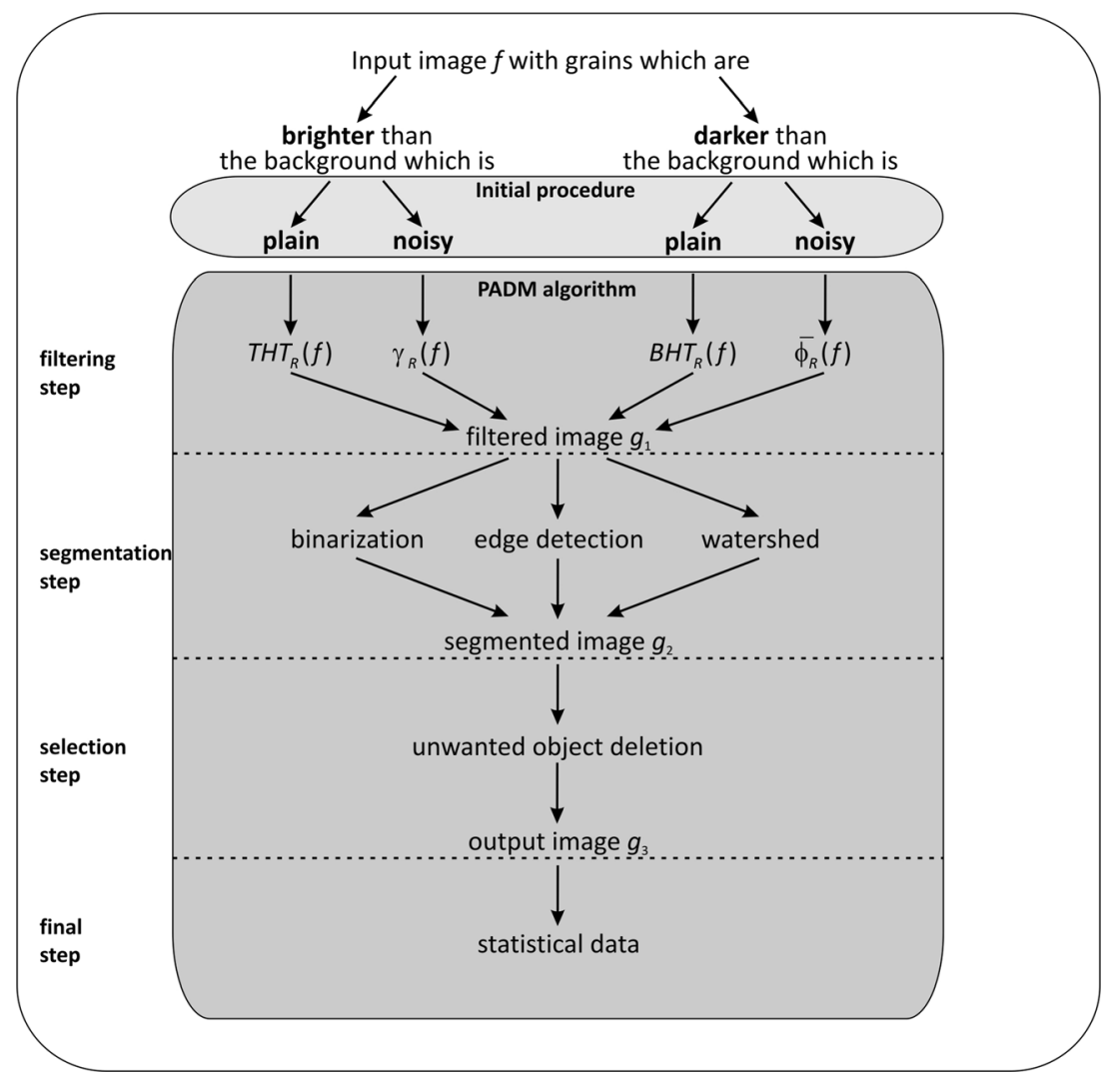


In the first step (filtering step), the background of an input image, $f$, is reduced or removed, and a filtered image $g_{1}$ is generated. If the $B G$ procedure returns a logical value false, and the studied grains are brighter than their surroundings, the filtering employs the top-hat transform $\left(\operatorname{THT}_{R}(f)\right)$. If the grains are darker than their surroundings the bottom-hat transform $\left(B H T_{R}(f)\right)$ is applied. Both transformations use a structuring element in the form of a disk of a range $R$, that is larger than the largest particles. If the background is noisy (the $B G$ procedure returns a logical value true), the opening procedure, $\gamma_{R}(f)$, is employed for objects whose values are larger than their surroundings or the negative of closing procedure $\bar{\phi}_{R}(f)$ is employed for objects whose values are smaller than their surroundings. Both procedures use a structuring element in the form of a disk of a range $R$, similar or larger than the background irregularities.

In the second step (segmentation step), a segmentation technique is applied to the filtered image. The segmentation step is different for each of the techniques and described below in more detail. As an output of this step a segmented binary image $g_{2}$ is calculated.

The third step (selection step) is related to removing unwanted objects from the segmented image. The applied selection procedures allow removing border particles and extracting objects that satisfy specific criteria, such as size (determined by the image resolution and the diameter of grains) or circularity (which depends on the studied grains characteristics), which also leads to reduction of segmentation artifacts. In this step, the final output image $g_{3}$, consisting of detected objects, is generated.

In the final step, the basic statistical analysis of the detected objects can be easily performed. The diameter, length, width, circularity and elongation of each particle as well as the particle size-distribution can be obtained and analyzed.

The binarization approach is the simplest and fastest. The steps of this technique are shown for two types of material. The first one is made of small dark grains located on a plain background (Fig. 4), and the latter of large bright grains located on a noisy background (Fig. 5).

In this approach, in the segmentation step, the filtered image, $g_{1}$, is binarized with the threshold operator $T_{[\alpha, \beta]}$, and all potential holes in features are filled (FILL). Thus this step returns a segmented image $g_{2}=F I L L\left[T_{[\alpha, \beta]}\left(g_{1}\right)\right]$. The binarization of studied images works well with Otsu's clustering threshold (Otsu 1979).

The edge detection approach can be used to detect separate grains with well-defined edges. In this work, for simplicity, the steps of this approach are shown only for the Martian material (Fig. 6). In the second step of this procedure, the
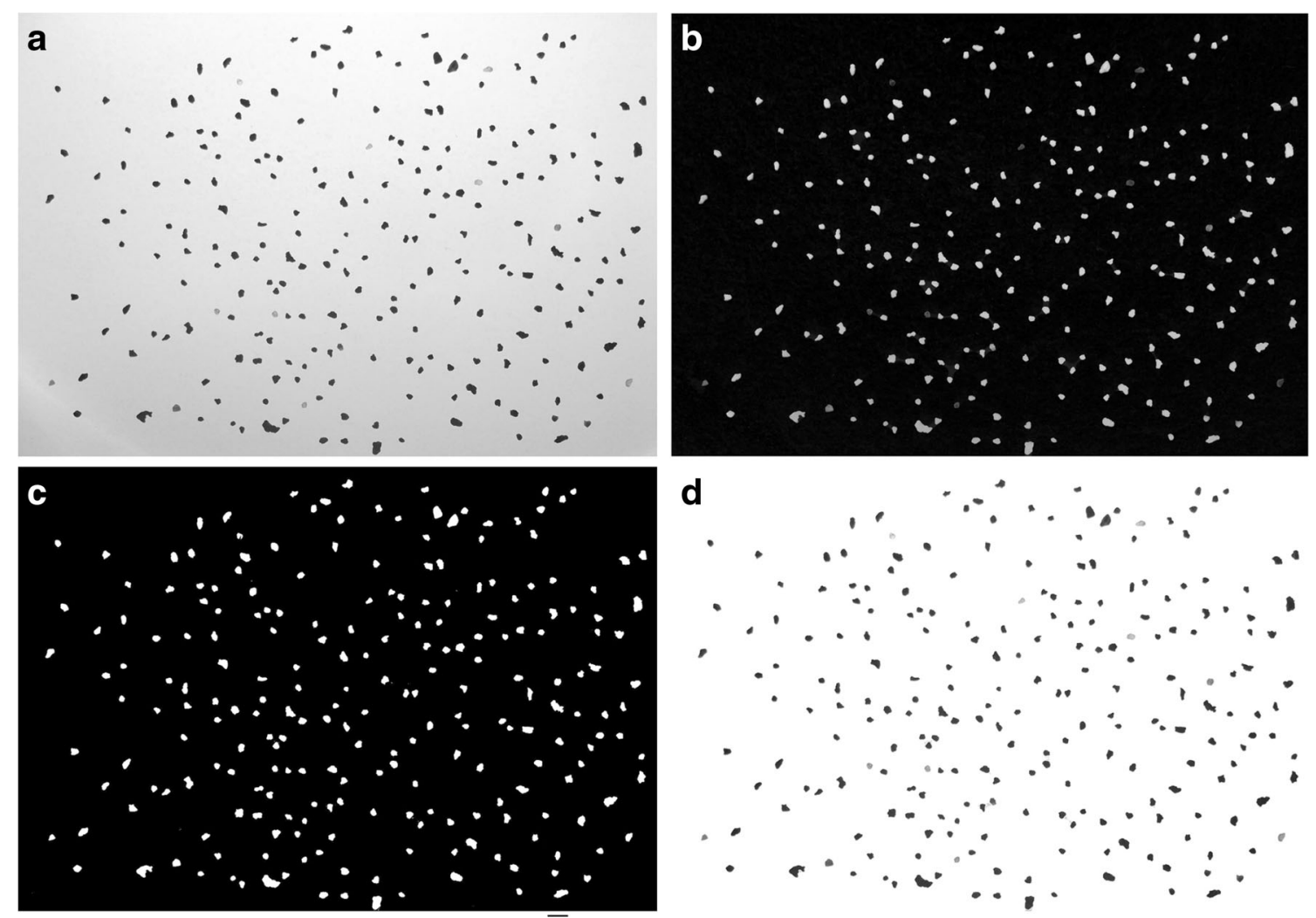

Fig. 4 The steps of the binarization technique for a terrestrial sample (Grand Falls dune field, Arizona, US) with a plain background. a Input image $f$. b Filtered image $g_{1}=B H T_{20}(f)$. c Segmented image $g_{2}=$

$F I L L\left[T_{[0.14,1]}\left(g_{1}\right)\right]$. d Detected particles. The input image $f$ is masked with the segmented image $g_{2}$. The image contains 229 grains in the range of 0.5 to $2.0 \mathrm{~mm}$. Image size is $1914 \times 1332 \mathrm{px}$ 

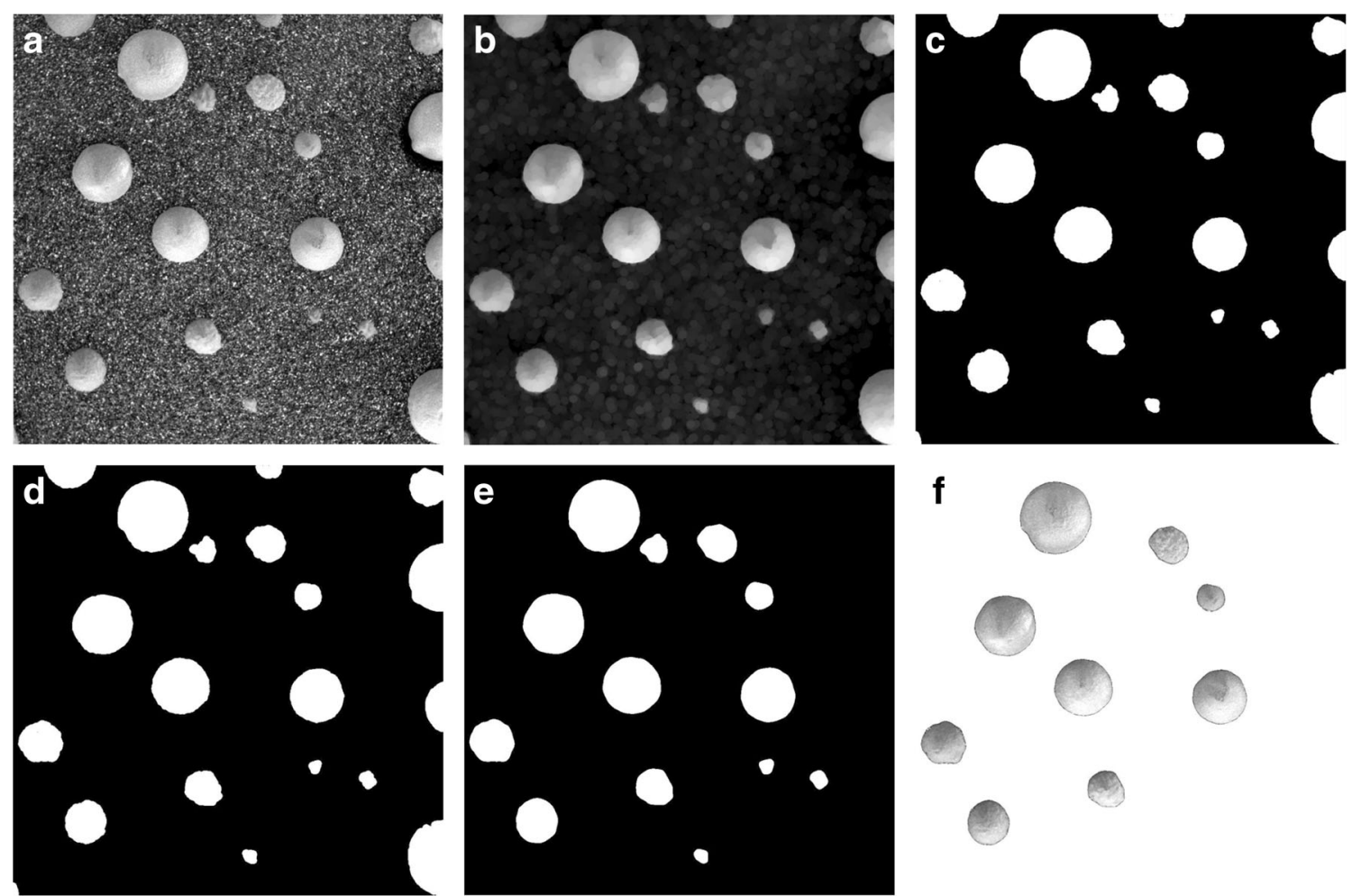

Fig. 5 The steps of the binarization technique for the MI image from Meridiani Planum (sol 1148, image ID: 1M230096684EFF81 O7P2956M2M1) with a noisy background. a Input image $f$. b Filtered image $g_{1}=\gamma_{8}(f)$. c Binarization of $g_{1}$ by Otsu's threshold. d Filling

operation of (c) results in the segmented image $g_{2}=F I L L\left[T_{[\mathrm{Otsu}]}\left(g_{1}\right)\right]$. e The extraction step. Border objects were removed automatically. f Detected particles. Four buried particles were removed manually, on the basis of human assessment
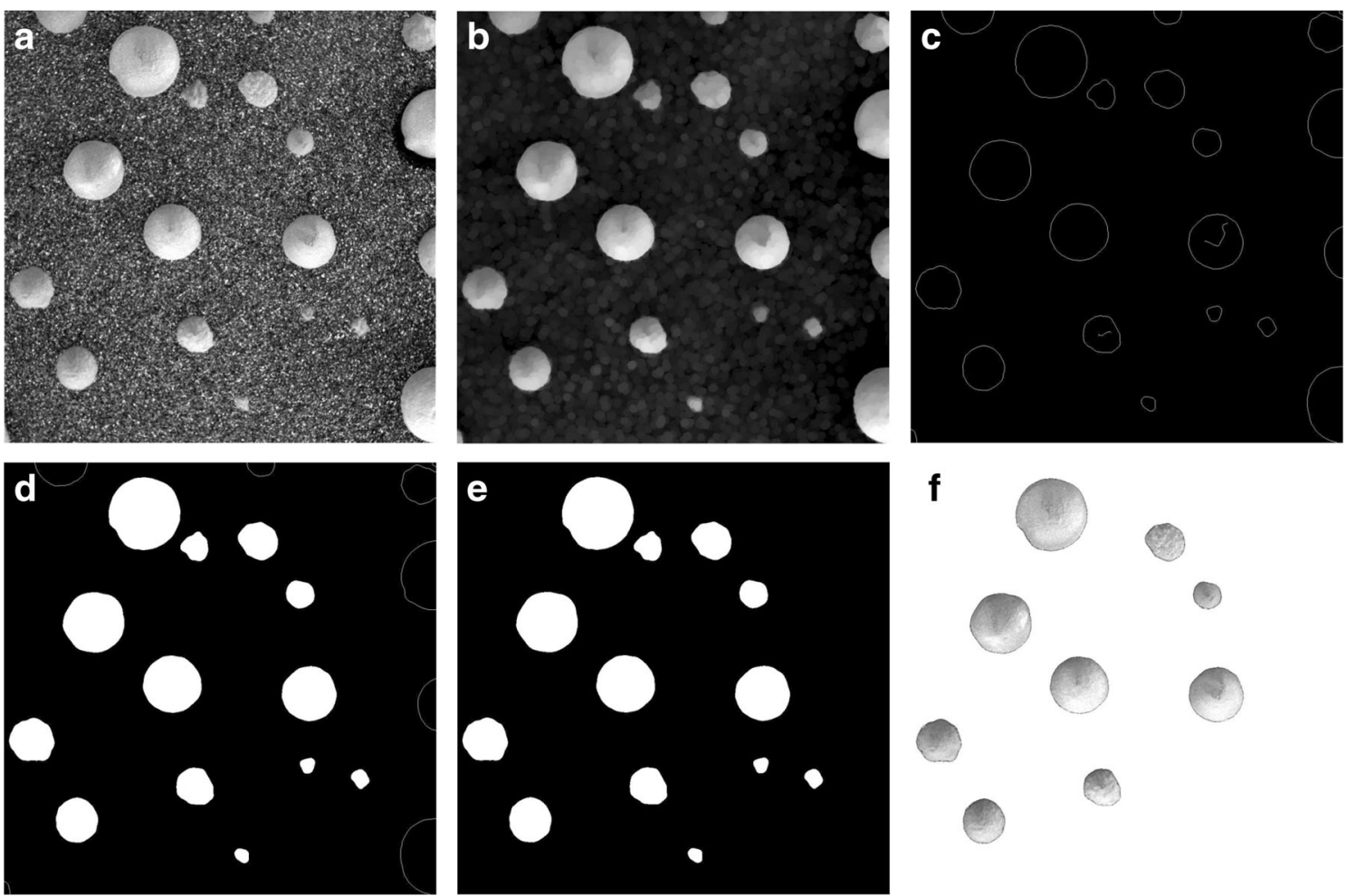

Fig. 6 The steps of the edge detection technique for the MI image from Meridiani Planum (sol 1148). a Input image $f$. b Opening of $f, g_{1}=\gamma_{8}(f)$. c Edge detection and thinning of $g_{1}, T H I N\left[E D_{9}\left(g_{1}\right)\right]$. d Filling contours of

(c). e The segmented image $g_{2}$ as a result of erosion and dilation of (d). $\mathbf{f}$ Detected particles. Four buried particles were removed manually, on the basis of human assessment 
Canny edge detection $(E D)$ algorithm is applied to the filtered image $g_{1}$ (Canny 1986), with a filter range $R$ similar to the grains size. Next, the thinning procedure (THIN) is used to thin the edges to one-pixel wide, and the computed outlines are filled. All artificial lines are removed using erosion $\varepsilon_{R}$ and dilation $\delta_{R}$ procedures with a square structuring element of a range $R=1 \mathrm{px}$. This technique returns the segmented image $g_{2}$ $=\delta_{1}\left[\varepsilon_{1}\left[\right.\right.$ FILL $\left[\right.$ THIN $\left.\left.\left.\left[E D_{9}\left(g_{1}\right)\right]\right]\right]\right]$.
The marker-controlled watershed segmentation technique (Soille 2002; Iwanowski 2009) can be used for touching as well as separated particles. In the watershed segmentation, the pixel value in an image is treated in a similar way as the elevation value in a topographic map, and boundaries between particles are determined in a similar way as boundaries between ridges. The steps of this approach are also shown only for the Martian material (Fig. 7). In the segmentation step, the reduced image, $g_{1}$,
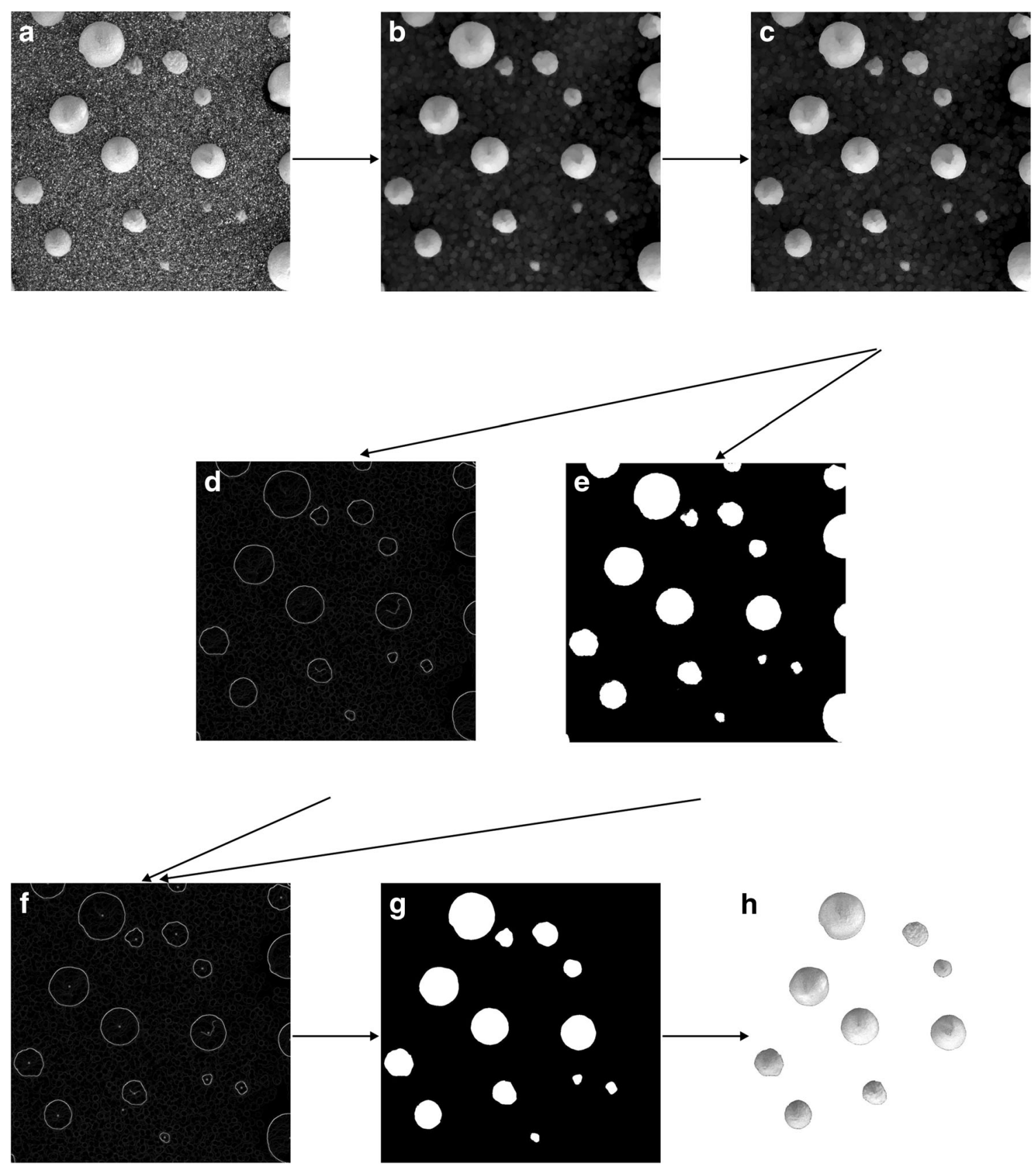

Fig. 7 The steps of the segmentation technique for the MI image from Meridiani Planum (sol 1148). a Input image $f$. b Opening of $f, g_{1}=$ $\gamma_{8}(f)$. c Sharpening of $g_{1}, g^{\prime}{ }_{1}=S H_{2}\left(g_{1}\right)$. d The $S F$ contours. e Binarization and filling of $g_{1}$ (f) Markers $M S$, shown as dots, with $S F$ contours. g Segmented image $g_{2}$ with removed border objects. h Detected particles. Four buried particles were removed manually, on the basis of human assessment 
can be sharpened $(S H)$ over a radius $R$, generating an enhanced image $g^{\prime}{ }_{1}=S H_{R}\left(g_{1}\right)$. In the enhance image, the contours of the grains can be detected by the Canny edge detection algorithm and added to the sharpened image to enhance grain boundaries (Fig. 7c). Next, two parallel processing sequences: gradient filtering and binarization are applied. In the first sequence, gradient filtering $(G F)$ with a kernel of pixel radius $R=1$ is performed. Gradient filtering generates a filtered image corresponding to the magnitude of the input image gradients. This allows for contours detection in the image and produces a segmentation function, $S F=G F_{R}\left[E D_{R}\left(g^{\prime}{ }_{1}\right)+g_{1}^{\prime}\right]$ (Fig. $\left.7 \mathrm{~d}\right)$. In the second sequence, the enhanced image $g^{\prime}{ }_{1}$ is binarized. Then, all holes in the binarized image are filled (Fig. 7e). From this image, the distance transform $(D T)$ is computed. The distance transform on the binary image connects each pixel with its distance to the nearest pixel whose value is 0 . This output image is submitted to Gaussian filtering $(G S)$ with a kernel of radius $R$. If the kernel radius is larger, the filtered image is smoother. The kernel of radius $R=15 \mathrm{px}$ can be used for medium grains (in relation to the image resolution). For large grains, $R$ can be up to $25 \mathrm{px}$, and for small grains (at the resolution limit) down to $1 \mathrm{px}$. Next, in the output image, the markers $(M S)$ of the particles are found using extended maxima detection (MAX) (see e.g. Soille 2002), $M S=$ $\operatorname{MAX}\left[G S_{R}\left[D T\left[F I L L\left[T_{[\alpha, \beta]}\left(g^{\prime}{ }_{1}\right)\right]\right]\right]\right]$ (Fig. 7f). A marker is a point in the image, which indicates that in the given place a particle is located. The $M S$ are employed to impose the segmentation function $S F$. The watershed transformation of the image, $W S\left(g_{1}{ }_{1}\right)$, is done by the Meyer method (Meyer and Beucher 1990) with 8 closest neighbors. Finally, the output is binarized with Otsu's threshold, producing the segmented image $g_{2}=T_{\text {[Otsu] }}\left[W S\left(g^{\prime}{ }^{\prime}\right)\right]$ (Fig. $7 \mathrm{~g}$ ).

The median diameters of the detected grains as well as the parameters for all the approaches are presented in the Appendix in Table 5.

\section{PADM algorithm testing in comparison with other methods}

The PADM algorithm was tested in comparison with other semi-automated and manual image analyses as well as with sieve analysis.

\section{Comparison with other automated image analyses}

Firstly, the PADM algorithm was tested on generated binary images representing various samples of particles. The same images were also analyzed using ImageJ, and Malvern Morphologi G3 system.

Next, to compare results for terrestrial samples, the PADM method was used along with the image analysis provided by the Malvern Morphologi G3 system. The Malvern Morphologi G3 system was used because it is more precise than ImageJ. For this study, three types of sands were employed. The first sample, HE, was composed of fine-grained beach basalt sand from a beach near Héraðssandur, Iceland. The second, GF, was acquired from the Grand Falls dune field, located ca. $70 \mathrm{~km} \mathrm{NE}$ of Flagstaff, Arizona, USA. The Grand Falls dune field deposit consists of fine to medium-grained quartz sand, and medium- to coarse-grained basalt sand (Hayward et al. 2010). The last sample, BD, was composed of finegrained quartz sand, and it was collected from a dune field site called the Błędów Desert, located ca. $60 \mathrm{~km} \mathrm{NW}$ of Krakow, Poland.

The Morphologi G3 system uses a semi-automated image analysis technique and provides particle size and shape information. The most important components of the device are the optical unit, digital camera, sample dispersion unit, and software. Using the system, one can receive high quality images of a studied sample along with its statistical analysis. Before analyzing, the samples were dispersed using a pulse of compressed air. For all sands, the same Standard Operating Procedure (SOP) and a $2.5 \times$ objective were used.

To compare the obtained results with the PADM algorithm, the images of the entire samples captured by the Morphologi $G 3$ camera were input into the algorithm.

\section{Comparison with sieve analysis}

To compare the results from the PADM algorithm with sieve analysis, three terrestrial samples were studied. The first two samples GF, and BD were the same as used in the previous comparison. The third sample, SO, consisted of coarse-
Table 1 The statistical results for an image containing 280 identical circular objects obtained by PADM, ImageJ and Morphologi G3

\begin{tabular}{lllll}
\hline & Number of grains & Mean $D[\mathrm{px}]$ & $\begin{array}{l}\text { Standard } \\
\text { deviation [px] }\end{array}$ & Mean circularity \\
\hline PADM (binarization) & 265 & 18.77 & 0.17 & 0.98 \\
PADM (edge detection) & 280 & 18.90 & 0.13 & 1.00 \\
PADM (watershed) & 263 & 18.84 & 0.14 & 0.97 \\
ImageJ & 266 & 18.74 & 0.17 & 0.95 \\
Morphologi G3 & 264 & 18.89 & 0.17 & 0.99
\end{tabular}


Table 2 Comparison of statistical results obtained by PADM (binarization) and Morphologi G3 from the same images of the terrestrial samples

\begin{tabular}{lllllll}
\hline Sample & Method & $\begin{array}{l}\text { Number of } \\
\text { grains }\end{array}$ & $\begin{array}{l}\text { Mean } D \\
{[\mathrm{~mm}]}\end{array}$ & $\begin{array}{l}\text { Median } D \\
{[\mathrm{~mm}]}\end{array}$ & $\begin{array}{l}\text { Standard deviation } \\
{[\mathrm{mm}]}\end{array}$ & $\begin{array}{l}\text { Mean } \\
\text { circularity }\end{array}$ \\
\hline GF & PADM & 605 & 0.23 & 0.16 & 0.22 & 0.81 \\
& Morphologi & 555 & 0.22 & 0.16 & 0.18 & 0.84 \\
HE & PADM & 578 & 0.33 & 0.32 & 0.10 & 0.88 \\
& Morphologi & 572 & 0.34 & 0.33 & 0.09 & 0.87 \\
BD & PADM & 747 & 0.22 & 0.19 & 0.10 & 0.90 \\
& Morphologi & 654 & 0.23 & 0.20 & 0.10 & 0.90 \\
\hline
\end{tabular}

grained basalt sand from Sólheimajökull glacier moraine, Iceland. Sieve analysis was performed with Fritsch sieves from $0.5 \mathrm{~mm}$ to $4.0 \mathrm{~mm}$. Sieving was conducted with mechanical shakers and hand shaking. The number of grains as well as the weight of each sample was established before and after employing sieve analysis. The number of grains was measured manually.

After sieve analysis, the images of all three samples were taken. All the images were captured with a Fuji FinePix S2995 camera. To test the algorithm efficiently, the images were taken in various conditions. Two kinds of lighting were applied: top light and back light. For dark grains, in order to reduce shadows, the back light was preferred, but it was not always possible, especially with images containing touching particles. In all cases, the images were to some extent unevenly illuminated. In many cases, grains were separated manually before processing, so that there were no touching particles in the images. To establish the size of grains, a ruler was used. The camera was leveled properly before taking each image.

\section{Analysis of MI and MAHLI images}

Finally, the PADM algorithm was evaluated with images taken by the Mars rovers. The statistical results from the PADM algorithm were also compared with the results obtained previously by manual methods with regard to some of the MI images of Meridiani Planum sediments (Weitz et al. 2006).

\section{Results}

Using the PADM algorithm, 76 terrestrial and Martian images were studied using all the approaches discussed above. The grain diameter, $D$, was defined as the diameter of a disk that has the same area (the equivalent disk). The circularity was calculated as: $4 \pi A / P^{2}$, where: $A$ is the area of a particle, and $P$ is the perimeter of a particle. Length, $L$, was assumed as the major axis of the best-fit ellipse (computed from moment invariants), and width, $W$, as the minor axis of the best-fit ellipse. Elongation was computed as $1-W / L$, and aspect ratio as $W / L$.
Fig. 8 Cumulative size distribution curves of the terrestrial samples: GF, HE, and $\mathrm{BD}$, obtained by PADM (binarization), and Morphologi G3 system (MORPH)

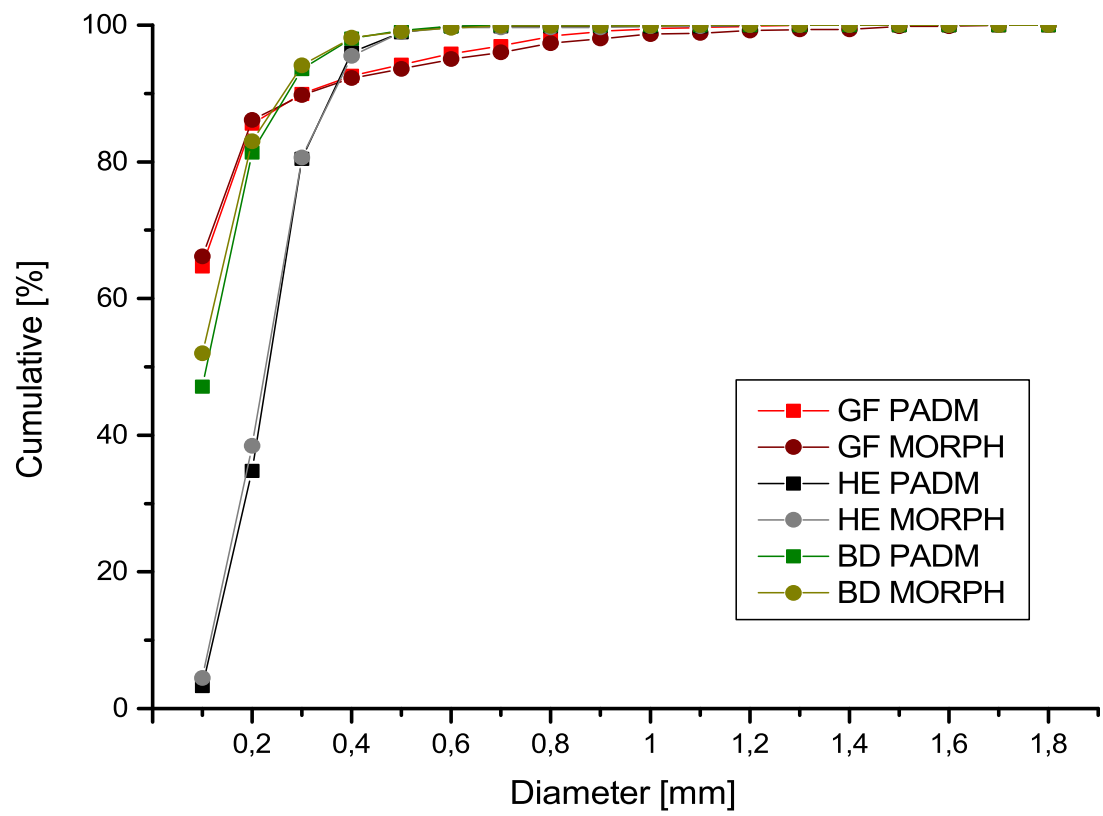


Table 3 Comparison of statistical results obtained by PADM (binarization) and sieve analysis for the terrestrial samples

\begin{tabular}{lllll}
\hline Sample & Method & Number of grains & Mean width [mm] & Standard deviation [mm] \\
\hline SO & PADM & 763 & 1.34 & 0.61 \\
& Sieve & & 1.28 & 0.59 \\
\multirow{2}{*}{ GF } & PADM & 933 & 1.24 & 0.53 \\
& Sieve & & 1.19 & 0.52 \\
BD & PADM & 758 & 0.89 & 0.31 \\
& Sieve & & 0.89 & 0.29 \\
\hline
\end{tabular}

\section{Comparison with ImageJ and Morphologi G3 system}

The statistical results of a grayscale image representing generated circular particles of almost the same size in comparison with the ImageJ and Morphologi G3 are presented in Table 1.

The comparisons of the morphological parameters and cumulative size frequency distributions for the images of the terrestrial samples computed by Morphologi G3 system and PADM algorithm are presented in Table 2 and Fig. 8. Only grains larger than $0.1 \mathrm{~mm}$ and smaller than $1.8 \mathrm{~mm}$ in diameter were analyzed. As difference between the results obtained from the different PADM techniques was not larger than 5\% for the studied samples, and Morphologi G3 system uses the binarization technique, only results from the PADM binarization approach are shown.

The Morphologi G3 system has some limitations. Firstly, using a $2.5 \times$ objective, the system cannot measures particles larger than $1 \mathrm{~mm}$. The second important limitation is a small field of view of the microscope. In the case of coarse-grained samples, many images are required to obtain a sufficient number of grains. Also, the method does not distinguish touching particles, so that it is crucial to apply precise dispersion before analyzing. In all samples, the PADM algorithm detected more grains than Morphologi G3, and it also detected larger and smaller particles at the same time.

\section{Comparison with sieve analysis}

Despite many differences, sieve analysis and image analysis can be compared (Fernlund 1998). An example of a comparison between these two methods for the terrestrial samples is presented in Table 3 and Fig. 9. In both cases, the statistical parameters were obtained using Folk and Ward definitions (Folk and Ward 1957). In those analyses, only grains larger than $0.5 \mathrm{~mm}$ were studied and the results were used only for methodological purposes and are not representative for the studied sediments. As all the PADM techniques produce similar results, solely those from the binarization approach are shown.
Fig. 9 Cumulative size distribution curves of the terrestrial samples: SO, GF, and BD, obtained by PADM (binarization), and sieve analysis

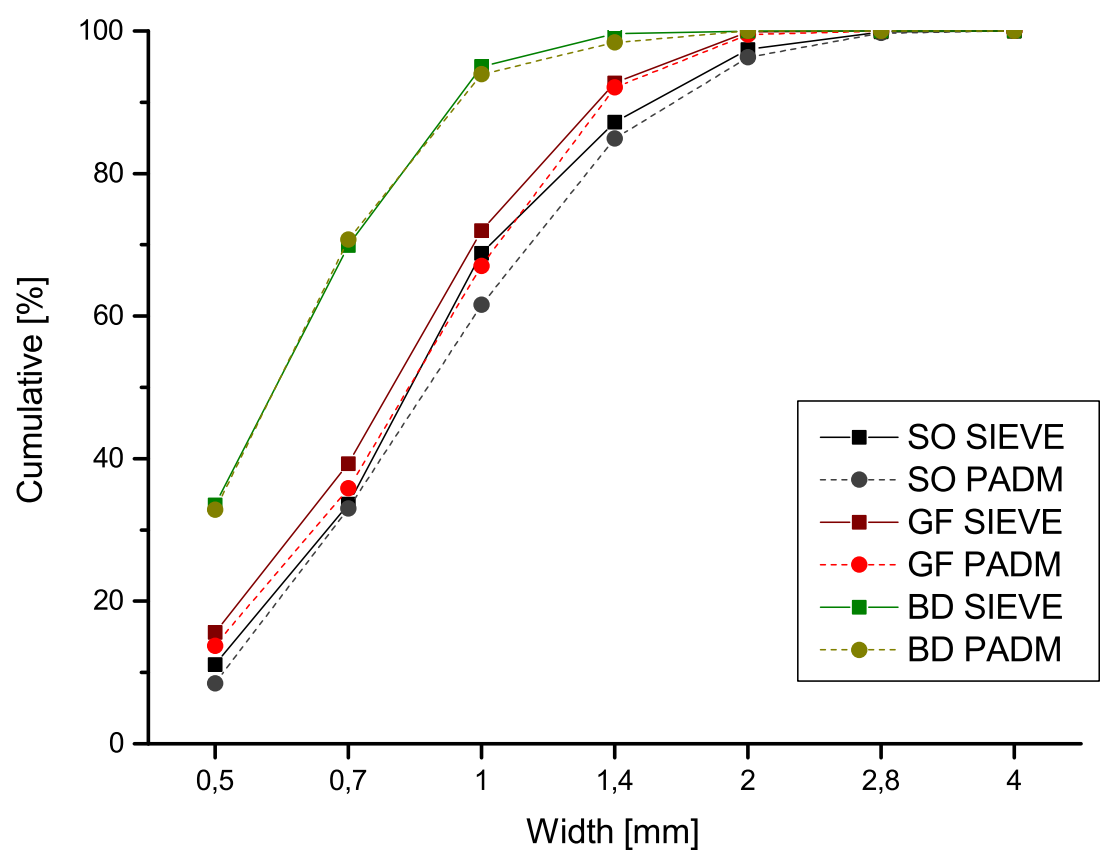




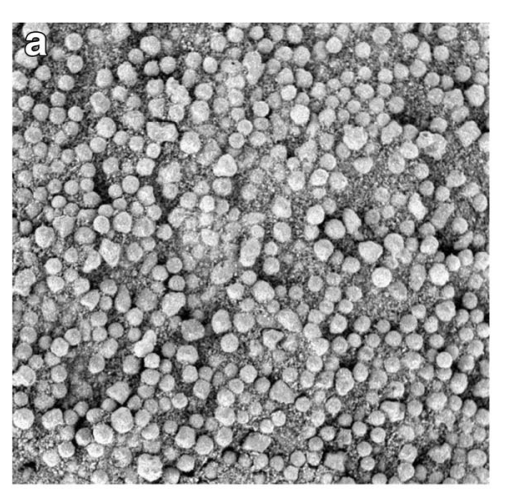

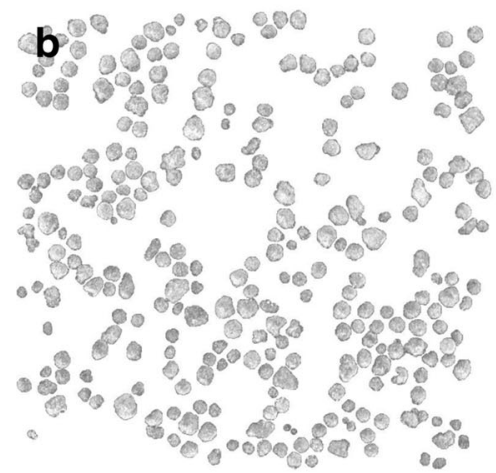

Fig. 10 Detection of bright streak grains from Meridiani Planum (sol 53, image ID: 1M132896293EFF06ASP2956M2M1) using different approaches. a Input image $f$. b Detected particles BIN. c Detected

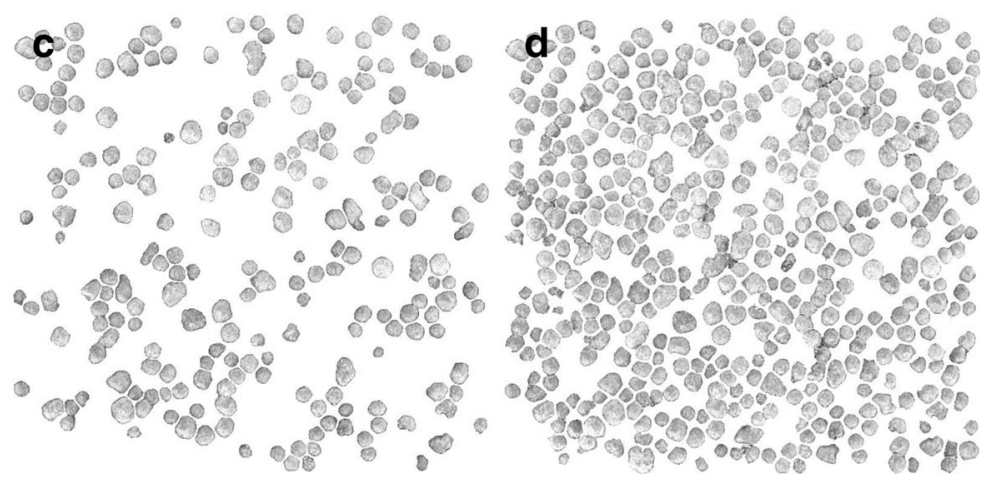

particles ED. d Detected particles WS. In all approaches unwanted objects were removed only automatically

mainly depends on the minor axis $c$. If $c$ is similar to $b$, we have the first extreme. If it is much smaller than $b$, we have the second extreme. As a result, we can assume that sieve analysis provides only some approximation of the intermediate axis of a grain, and it can, to some extent, be compared only to the width of the grain, $W$. Moreover, sieve analysis is destructive for fragile grains. The basalt sands in the samples GF and SO were characterized by sharp edges and were vulnerable for abrasion. After sieve analysis, in both cases destruction of
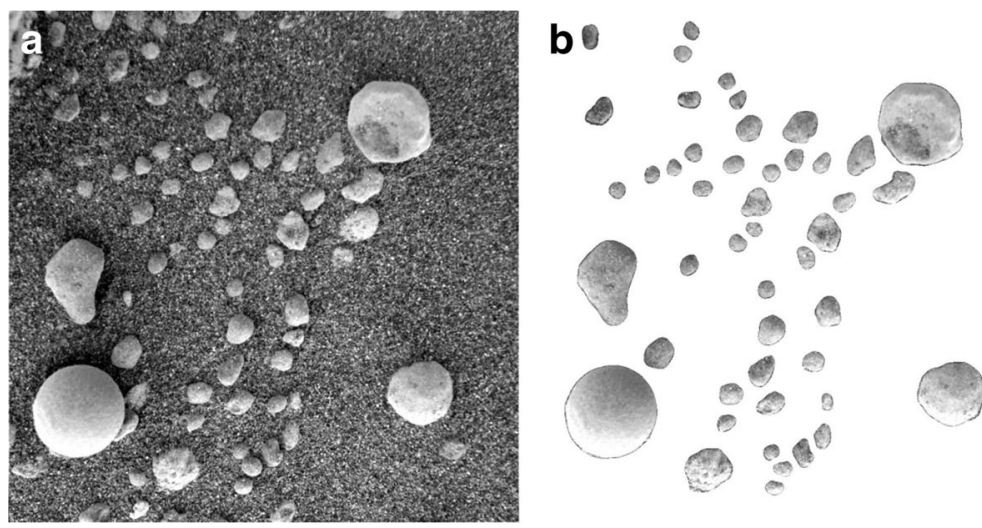

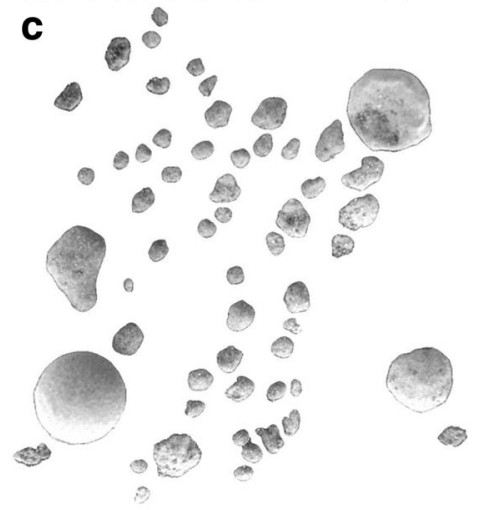

range $R=12 \mathrm{px}$, all the other grains with a structuring element of a range $R=9$. In both sequences, unwanted objects such as small or border particles were removed automatically whereas objects such as partially buried grains, were removed manually
Planum (sol 19) using the edge detection and the watershed. a Input image $f$. b Detected particles ED. c Detected particles WS. The largest grain, in the lower left corner, was detected with a structuring element of a 
Fig. 12 Detection of 458 particles from Meridiani Planum (sol 368) using the watershed sequence with the same parameters as in Fig. 10. a Input image $f$. b Detected particles. Unwanted objects were removed only automatically

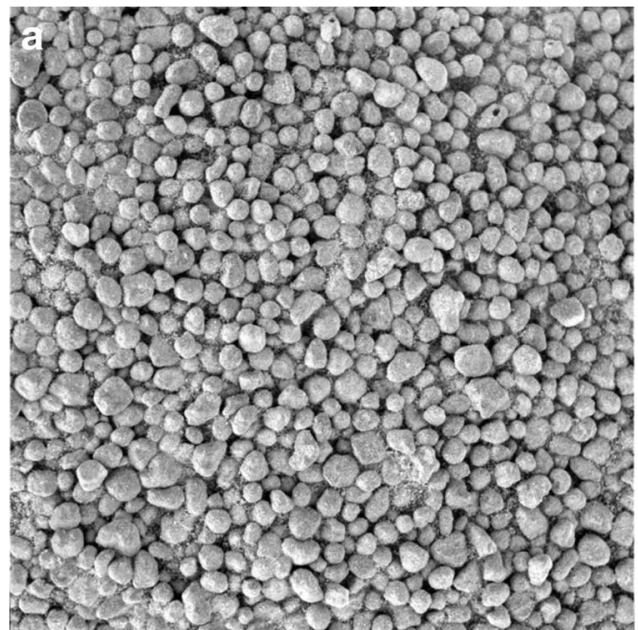

larger grains was clearly visible. In case of GF, ca. 5\% wt of the material was abraded. Also, in those cases during sieving many larger grains blocked openings, so that smaller grains were less likely to pass through a sieve. Therefore, the sieving process took more time than in the case of the BD sample. Next, sieve analysis normally gives the mass of material, not the number of grains. If we would like to establish which particles are the most frequently transported, then we will ask which class is the most numerous and not the most massive. These two parameters may not be related to the same class, especially as we have grains of various origins in the sample. In the case of GF sample, the most massive was class $0.7-1.0 \mathrm{~mm}$, and the most numerous $0.5-0.7 \mathrm{~mm}$. Finally, sieve analysis does not allow determining the shape of grains.

2D image analysis methods also have some shortcomings. The first one is associated with the number of grains that are analyzed. In sieve analysis, tens of thousands of grains are studied. In an image analysis, rather thousands or hundreds

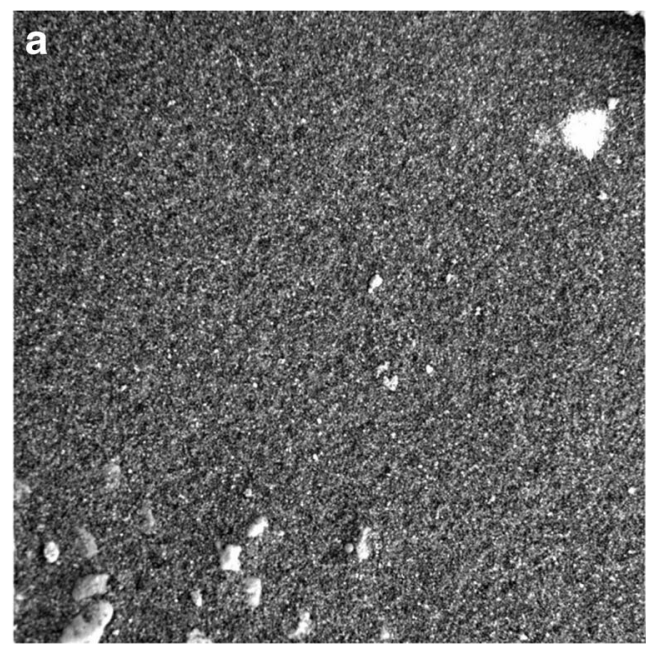

Fig. 13 The detection of small bright particles in the MI image from Meridiani Planum (sol 165, image ID: 1M142830062EFF3221 $\mathrm{P} 2976 \mathrm{M} 2 \mathrm{M} 1$ ) using the binarization sequence. a Input image $f$. b of grains. However, the significant tests indicated that the obtained mean width is valid for all studied terrestrial samples with a statistical significance level of 0.05 . Also, this method does not provide information on the minor axis, $c$.

\section{Analysis of MI and MAHLI images}

Using the PADM algorithm, 43 images taken by the Mars rovers: Spirit, Opportunity, and Curiosity, were analyzed. Particles truncated by an image or partly buried were omitted as their size and shape cannot be fully determined. Examples of detection are presented for six types of material: bright streak deposit (Fig. 10), spherules (Fig. 11), ripple crest sand (Fig. 12), fragments of larger grains (Fig. 13), dust aggregates (Fig. 14), and dune sand (Fig. 15). The first four images were taken in Meridiani Planum, the fifth in Gusev Crater, and the last one in Gale Crater. A comparison between the PADM results and those from a manual method for the presented

b

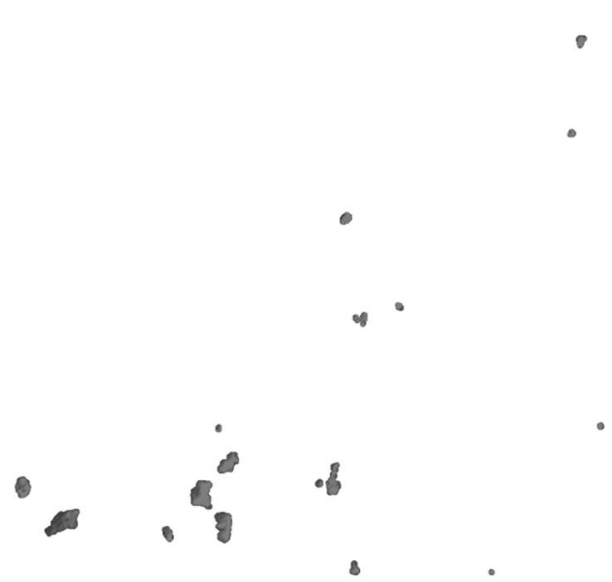

Detected particles. 17 bright grains in the range of diameter from 0.30 to $1.30 \mathrm{~mm}$ were identified. A few unwanted objects, such as partially buried particles and a bright spot, were deleted manually 

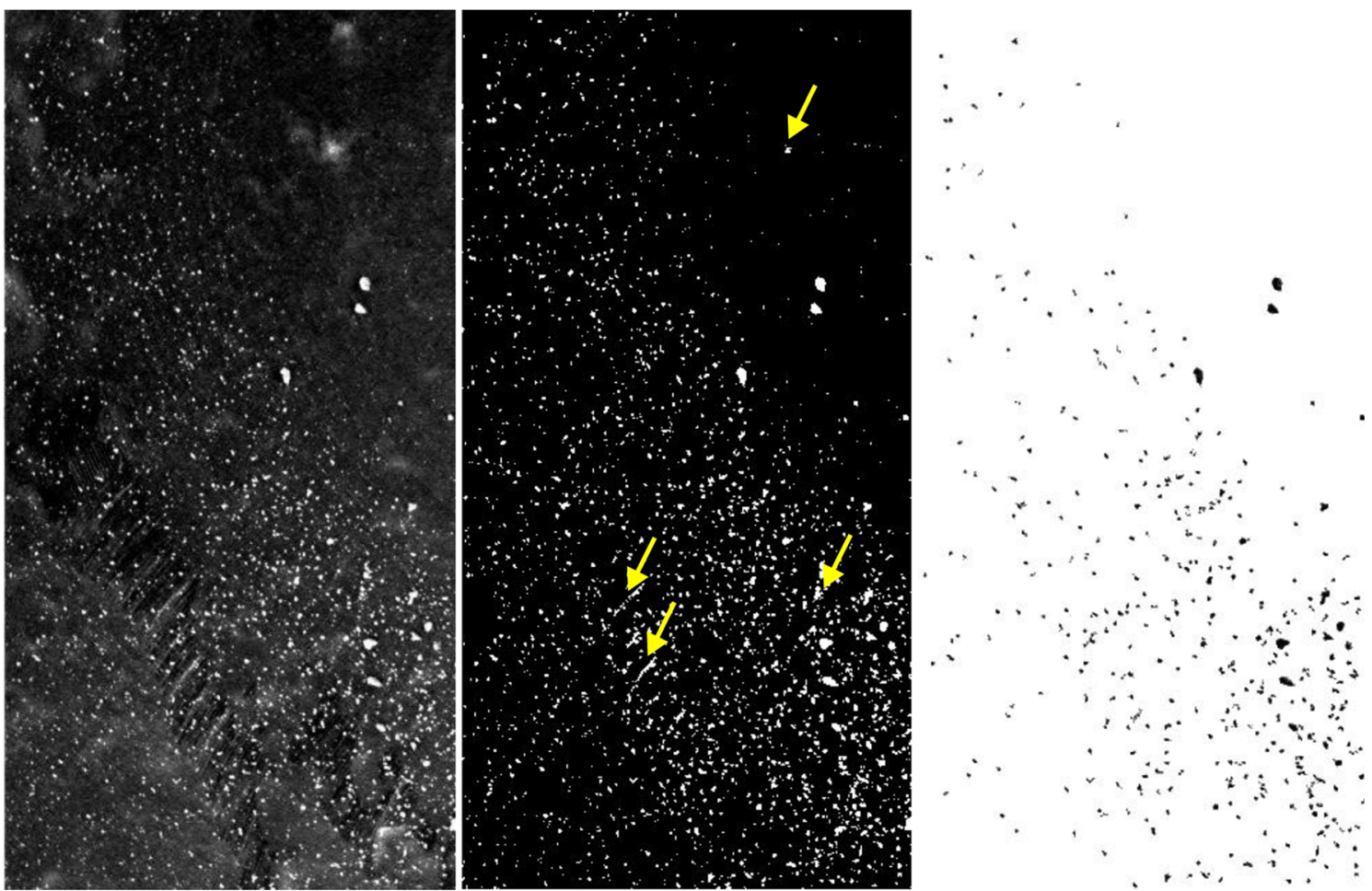

Fig. 14 Detection of 344 dust aggregates from Gusev Crater (sol 79) by PADM (binarization). The largest grain is ca. $0.30 \mathrm{~mm}$ in diameter. The arrows indicate unwanted objects that were removed manually

samples of Meridiani Planum deposits are shown in Table 4. All the parameters used in the analyses with the PADM algorithm are presented in Appendix Table 5.

The errors of the method can be divided into two groups. The first group is associated with inaccuracies in the detection, e.g. undetected grains, badly segmented (e.g. over- or undersegmented) images. These errors can be reduced by manual correction to some extent. The detection inaccuracy can also be associated with non-grain features that are present in some of the images. For example, in the image from Spirit rover acquired on sol 79 (Fig. 14), there are some bright linear

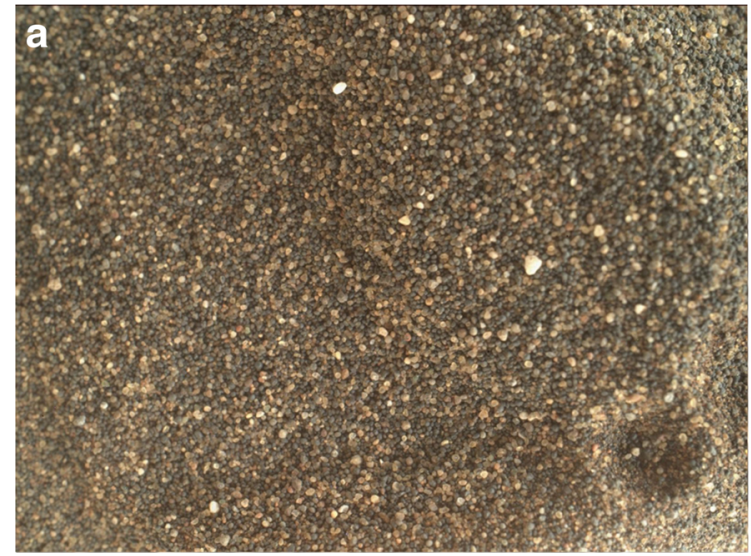

Fig. 15 Detection of more than 3000 bright sand grains from Gale Crater (sol 1242, image ID: 1242MH0005740000403709R00_D14761) by PADM (watershed). a Input image $f$. b Detected particles. The largest grain is ca. $0.58 \mathrm{~mm}$ in diameter. Only bright grains were studied, as it structures and one bright spot, which are detected by the algorithm. However, in such situations the manual correction is related to ca. $1 \%$ of the detected objects. Also for images containing numerous grains, the detection inaccuracy does not significantly influence the result, so that manual correction is not necessary (Fig. 10). The second group is related to a systematic error of the given technique. This error must be taken into consideration in any statistical analysis.

The binarization technique detects often smaller number of grains for a given image than the watershed but larger than the edge detection approach. The systematic error of this technique

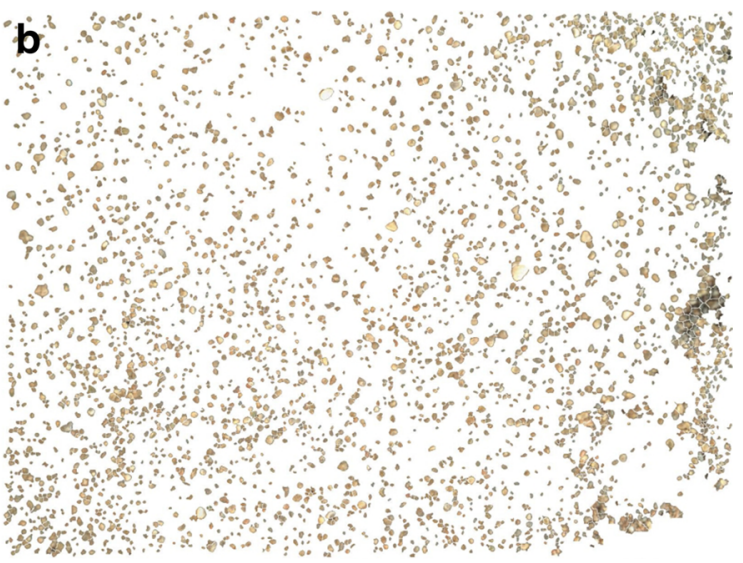

was difficult to distinguish dark particles from holes (shadows) among grains. The right side of the image is out of focus, and the result for this part of the image is therefore affected. Unwanted objects were removed only automatically 
Table 4 Comparison between result from the manual method (Weitz et al. 2006) and the PADM algorithm for three Martian samples from Meridiani Planum (BIN - binarization, ED - edge detection, WS - watershed)

\begin{tabular}{|c|c|c|c|c|c|c|}
\hline Sample & Method & Number of grains & Mean $L[\mathrm{~mm}]$ & Median $L[\mathrm{~mm}]$ & Mean aspect ratio & Mean circularity \\
\hline \multirow[t]{4}{*}{ sol 53 (Fig. 10) } & BIN & 237 & 0.99 & 0.85 & 0.80 & 0.88 \\
\hline & ED & 203 & 0.94 & 0.86 & 0.86 & 0.92 \\
\hline & WS & 435 & 0.93 & 0.87 & 0.83 & 0.86 \\
\hline & Manual & 263 & 0.97 & 0.89 & 0.88 & 0.95 \\
\hline \multirow[t]{3}{*}{ sol 19 (Fig. 11) } & ED & 47 & 1.86 & 1.48 & 0.80 & 0.89 \\
\hline & WS & 57 & 1.83 & 1.46 & 0.78 & 0.85 \\
\hline & Manual & 51 & 1.76 & 1.46 & 0.77 & 0.90 \\
\hline \multirow[t]{2}{*}{ sol 368 (Fig. 12) } & WS & 458 & 1.27 & 1.19 & 0.77 & 0.79 \\
\hline & Manual & 246 & 1.34 & 1.28 & 0.81 & 0.92 \\
\hline
\end{tabular}

is related to the value of the threshold, and it is less than $5 \%$ in the grain size determination. In cases of images with some touching grains, the mean values (but usually not the median values) of the grain size can be overestimated, as some grains may not be separated (Fig. 10b). For images with a noisy background, the error associated with the shape determination can occur, and in such cases the mean circularity is often underestimated (Table 4). From all the presented approaches only binarization technique, due to its simplicity, works fine with low-resolution images (in which the size of the studied particles is only slightly above the image resolution).

The detection accuracy (the ratio of well detected grains to all detected grains) in the edge detection case is greater than in the binarization case. Yet, the number of grains detected by the edge detection approach is often smaller, as this technique detects only grains with well-distinguished edges. The statistical error of this technique depends on the size of the Gaussian kernel, and it is less than $4 \%$. In addition, the edge detection procedure tends to shift the center of the contours towards the grain exterior. This leads to systematic error that is ca. $2 \%$ in the grain size. This approach is especially good for measuring the shape of grains.

The marker-controlled segmentation accuracy depends mainly on the precision of the markers determination. This approach detects the largest percent of grains. Yet, the detection accuracy is smaller than that obtained from the edge detection technique. The statistical error of this procedure is up to ca. $7 \%$ in the grain size. In cases of overlapping or touching grains, the contours are more difficult to determine using the watershed approach, and their circularity value is often underestimated (Table 4).

The accuracy of the fitting of the grain contours (as compared with the manual assessment) for the studied set of 43 Martian images with the best-fit procedure of the PADM algorithm ranges from $85 \%$ to $100 \%$, with the average ca. $94 \%$. (For two images the accuracy was not determined due to high number of small grains). Yet, the inaccuracies with contours' fitting only slightly influence the statistical results of the size of the grains. Therefore, the detection accuracy does not influence the statistical analysis of the grain diameter more than the systematic error of the method.

\section{Conclusions}

The PADM algorithm in comparison to the algorithm presented in the work of Karunatillake et al. (2014) is much simpler. Using PADM we can employ three different techniques of segmentation, and it is not necessary to study the foreground separately. The number of parameters in the PADM algorithm is also lower. As the PADM algorithm is very fast, it is not necessary to convert color images to grayscale images, and information in all channels is available.

The PADM algorithm can be used with a graphical user interface (GUI). The GUI was prepared with the Wolfram Mathematica Manipulate module. For similar images, the automated mode of the PADM algorithm (in which all the necessary parameters are set) can be employed. Using the GUI, the automated analysis of an image whose size is similar to the size of the MI image takes ca. $3 \mathrm{~s}$ with a typical laptop. This analysis includes the image segmentation, the computation of the number of grains, the median and the mean diameter, and plotting granulometry curves. The semi-automated analysis, in which the user must adjust the parameters, takes from a few seconds to a few minutes - depending on the image, segmentation technique, and the expecting precision of the results.

The developed semi-automatic method allows fast granulometric analysis of various deposits. It provides similar results to sieve analysis or those obtained by manual methods. Furthermore, it allows studying the shape of grains, does not have any limitation on the size of grains, and permits the separation of touching particles. The systematic error of the method does not exceed $7 \%$ in the grain size.

Acknowledgments The author thanks James Zimbelman, Marsha Presley, and Leszek Nowak for suggestions and feedback on the readability of the manuscript. 


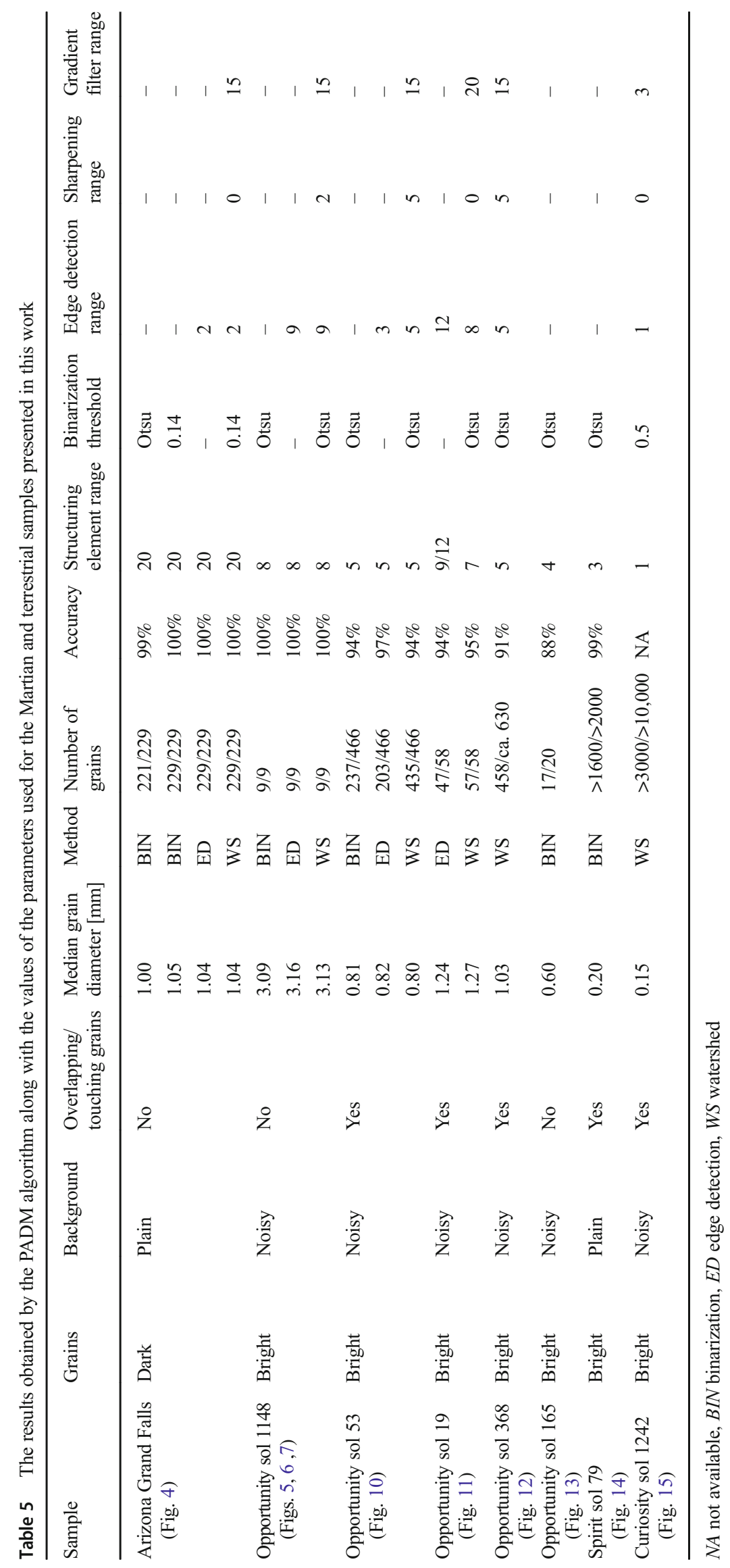


Open Access This article is distributed under the terms of the Creative Commons Attribution 4.0 International License (http:// creativecommons.org/licenses/by/4.0/), which permits unrestricted use, distribution, and reproduction in any medium, provided you give appropriate credit to the original author(s) and the source, provide a link to the Creative Commons license, and indicate if changes were made.

\section{References}

Borowka RK (1980) Współczesne procesy transportu i sedymentacji piasków eolicznych oraz ich uwarunkowania i skutki na obszarze wydm nadmorskich. Prace Komisji Geograficzno-Geologicznej. PWN, Warszawa, p 126

Canny J (1986) A computational approach to edge detection. IEEE Trans Pattern Anal Mach Intell 8(6):679-698

Edgett K et al (2012) Curiosity's Mars hand lens imager (MAHLI) investigation. Space Sci Rev 170(1-4):259-317. https://doi.org/10.1007/ s11214-012-9910-4

Fernlund JMR (1998) The effect of particle form on sieve analysis: a test by image analysis. Eng Geol 50(1-2):111-124. https://doi.org/10. 1016/S0013-7952(98)00004-0

Folk RL, Ward WC (1957) Brazos River bar: a study in the significance of grain size parameters. J Sediment Petrol 27(1):3-26. https://doi. org/10.1306/74D70646-2B21-11D7-8648000102C1865D

Friedman GM (1967) Dynamic processes and statistical parameters compared for size frequency distribution of beach and River Sands. J Sediment Petrol 37(2):327-354

Fu K, Mui J (1981) The survey on image segmentation. Pattern Recogn 13(1):3-16. https://doi.org/10.1016/0031-3203(81)90028-5

Geissler PE et al (2008) First in situ investigation of a dark wind streak on Mars. J Geophys Res 113:E12S31. https://doi.org/10.1029/ 2008JE003102

Hayward RK et al (2010) Second internationally dune workshop. LPI Contribution No 1552:27-28

Herkenhoff KE et al (2003) Athena microscopic imager investigation. J Geophys Res 108:8065. https://doi.org/10.1029/ 2003JE002076,E12.

Herkenhoff KE et al (2006) Overview of the microscopic imager investigation during spirit's first 450 sols in Gusev crater. J Geophys Res 111:E02S04. https://doi.org/10.1029/2005JE002574

Iwanowski M (2009) Metody morfologiczne w przetwarzaniu obrazów cyfrowych. Exit, Warszawa, p 255

Jerolmack DJ et al (2006) Spatial grain size sorting in eolian ripples and estimation of wind conditions on planetary surfaces: application to Meridiani Planum, Mars. J Geophys Res 111:E12S02. https://doi. org/10.1029/2005JE002544

Karunatillake S, McLennan SM, Herkenhoff KE, Husch JM, Hardgrove C, Skok JR (2014) A martian case study of segmenting images automatically for granulometry and sedimentology, part 1: algorithm. Icarus 229:400-407. https://doi.org/10.1016/j.icarus.2013. 10.001
Lira C et al (2010) Full granulometric curves of Meridiani soils, LPSC XLI, 2043

Matheron G (1967) Éléments pour une théorie des milieux poreux. Masson et Cie, Paris, p 168

Meyer F, Beucher S (1990) Morphological segmentation. J Vis Commun Image Represent 1(1):21-45. https://doi.org/10.1016/10473203(90)90014-M

Minitti ME, Kah LC, Yingst RA, Edgett KS, Anderson RC, Beegle LW, Carsten JL, Deen RG, Goetz W, Hardgrove C, Harker DE, Herkenhoff KE, Hurowitz JA, Jandura L, Kennedy MR, Kocurek G, Krezoski GM, Kuhn SR, Limonadi D, Lipkaman L, Madsen MB, Olson TS, Robinson ML, Rowland SK, Rubin DM, Seybold C, Schieber J, Schmidt M, Sumner DY, Tompkins VV, van Beek JK, van Beek T (2013) MAHLI at the Rocknest sand shadow: science and science-enabling activities. J Geophys Res Planets 118(11): 2338-2360. https://doi.org/10.1002/2013JE004426

Moiola RJ, Weiser D (1968) Textural parameters: an evaluation. J Sediment Petrol 38(1):45-53

Otsu N (1979) A threshold selection method from gray-level histograms. IEEE Trans Syst Man Cybern 9(1):62-66. https://doi.org/10.1109/ TSMC.1979.4310076

Soille P (2002) Morphological image analysis: principles and applications. Springer-Verlag, Berlin, p 391

Squyres SW, Arvidson RE, Bell JF 3rd, Brückner J, Cabrol NA, Calvin W, Carr MH, Christensen PR, Clark BC, Crumpler L, Marais DJ, d'Uston C, Economou T, Farmer J, Farrand W, Folkner W, Golombek M, Gorevan S, Grant JA, Greeley R, Grotzinger J, Haskin L, Herkenhoff KE, Hviid S, Johnson J, Klingelhöfer G, Knoll AH, Landis G, Lemmon M, Li R, Madsen MB, Malin MC, McLennan SM, McSween HY, Ming DW, Moersch J, Morris RV, Parker T, Rice JW Jr, Richter L, Rieder R, Sims M, Smith M, Smith P, Soderblom LA, Sullivan R, Wänke H, Wdowiak T, Wolff M, Yen A (2004) The opportunity Rover's Athena science investigation at Meridiani Planum, Mars. Science 306(5702):1698-1703. https:// doi.org/10.1126/science. 1106171

Sullivan R et al (2008) Wind-driven particle mobility on Mars: insights from Mars Exploration Rover observations at "El Dorado" and surroundings at Gusev Crater. J Geophys Res 113:E06S07. https://doi. org/10.1029/2008JE003101

Visher GS (1969) Grain-size distributions and depositional processes. J Sediment Petrol 39:1074-1106

Weitz CM et al (2006) Soil grain analyses at Meridiani Planum, Mars. J Geophys Res 111:E12S04. https://doi.org/10.1029/2005JE002541

Yingst RA, Kah LC, Palucis M, Williams RME, Garvin J, Bridges JC, Bridges N, Deen RG, Farmer J, Gasnault O, Goetz W, Hamilton VE, Hipkin V, Jensen JK, King PL, Koefoed A, le Mouélic SP, Madsen MB, Mangold N, Martinez-Frias J, Maurice S, McCartney EM, Newsom H, Pariser O, Sautter VH, Wiens RC (2013) Characteristics of pebble- and cobble-sized clasts along the curiosity rover traverse from Bradbury landing to Rocknest. J Geophys Res Planets 118(11):2361-2380. https://doi.org/10.1002/2013JE004435

Yingst RA et al (2008) Morphology and texture of particles along the Spirit rover traverse from sol 450 to sol 745. J Geophys Res 113: E12S41. https://doi.org/10.1029/2008JE003179 